\title{
Gesticulation Based Smart Surface with Enhanced Biometric Security Using Raspberry Pi
}

\author{
R.Gayathri ${ }^{1}$, E.Roshith ${ }^{2}$, B.Sanjana ${ }^{2}$, S. Sanjeev Kumar ${ }^{2}$, R.Shyamala $^{2}$ \\ ${ }^{1,2}$ Department of Electronics and Communication \\ Sri Venkateswara College of Engineering \\ Sriperumbudur,Tamil Nadu,602117, India
}

\begin{abstract}
This system converts any flat surface into a smart gesture recognition system with the help of digital image processing. The hand movements are recognized using Open $C V$ software and it is processed in Raspberry $P i$ and the actuating signals are given to Arduino to control a number of infrared based devices and to visual studio to control mouse pointer on computer. The privacy is enhanced with the help of fingerprint Biometrics, and the requirement of flat surface makes it possible to capture the movement with greater accuracy. Here we can control many devices from anywhere inside the house. The Biometric will allow only restricted personnel to access the devices and a time-out feature along with it will further enhance the privacy.
\end{abstract}

Keywords - Image processing, Open CV, Raspberry Pi, Arduino, Biometrics.

\section{Introduction}

The methods to control various electronic devices have evolved from tuning knobs to the currently prevailing IR remotes. Gesture recognition builds a richer bridge between human and computers than the conventional GUI and remotes. Using a number of input devices to control various systems can be annoying and tedious. Universal remotes solve this problem to an extent but the line of sight is required. Controlling mouse pointer along with the IR devices obviates the line of sight requirement.

Here we are using vision based hand gesture recognition. The inputs are taken from the Raspberry Pi camera. The image is processed using Open CV software in Raspberry $\mathrm{Pi}$ and the signal is given to Arduino via serial communication. The same output from the Raspberry $\mathrm{Pi}$ is fed into a windows based system and with the help of visual studio the mouse pointer is controlled. In order to enhance the privacy of the system we have included bio-metric recognition. The bio-metric is implemented using Arduino.

\section{Literature Survey}

Hand gesture recognition systems are mainly created to develop an environment where human computer interactions can be done efficiently with much ease. Many researchers have been carried out and lot of works have been done in this field by various researchers.There are methods to control various devices through E-mail, gesture recognizing pendants, hats with cameras etc. A number of approaches are there for the recognition of the gesture and processing them. Kinematic model infers the pose of the palm and the joint angles. It is ideal for realistic interactions in virtual environments [1, 2]. View based approach models the gestures as sequence of images. [3]Low level based approaches are robust to noise and the images can be extracted quickly [4]. Zigbee based systems along with wireless networks have been used in home automation for implementing flexible wireless architecture [5].

\section{Proposed System}

The figure 1.1 describes the working. It starts with the bio-metric authentication of the finger print [6]. Arduino is used for interfacing the fingerprint sensor. The bio-metric authentication has three steps enrolment, identification, and decision. Here we can store up to 200 fingerprints. If the authentication is done we can access the device which is constituted by two main parts

1.Raspberry pi

2.Raspberry pi camera.

The camera is positioned to capture the hand movement. The Raspberry Pi Camera captures the hand movement and the image is processed by using the Open CV software. The outline of the hand is mapped and the movement of the finger is recognized by means of the change in the outline. The complete processing is done using Raspberry Pi and the output from that is given as a control signal for actuating a number of devices at home and it is also used to move the cursor on the computer screen. To interface with the various devices ATMega-328 Microcontroller is used and for the mouse pointer $\mathrm{g}$ Visual Studio is used. For the devices like television, air conditioner, etc. For the proposed hardware implementation IR protocol and an IR to RF extender are used to increase the range of the signal so that we could control the devices without actually coming in line of sight with them. 


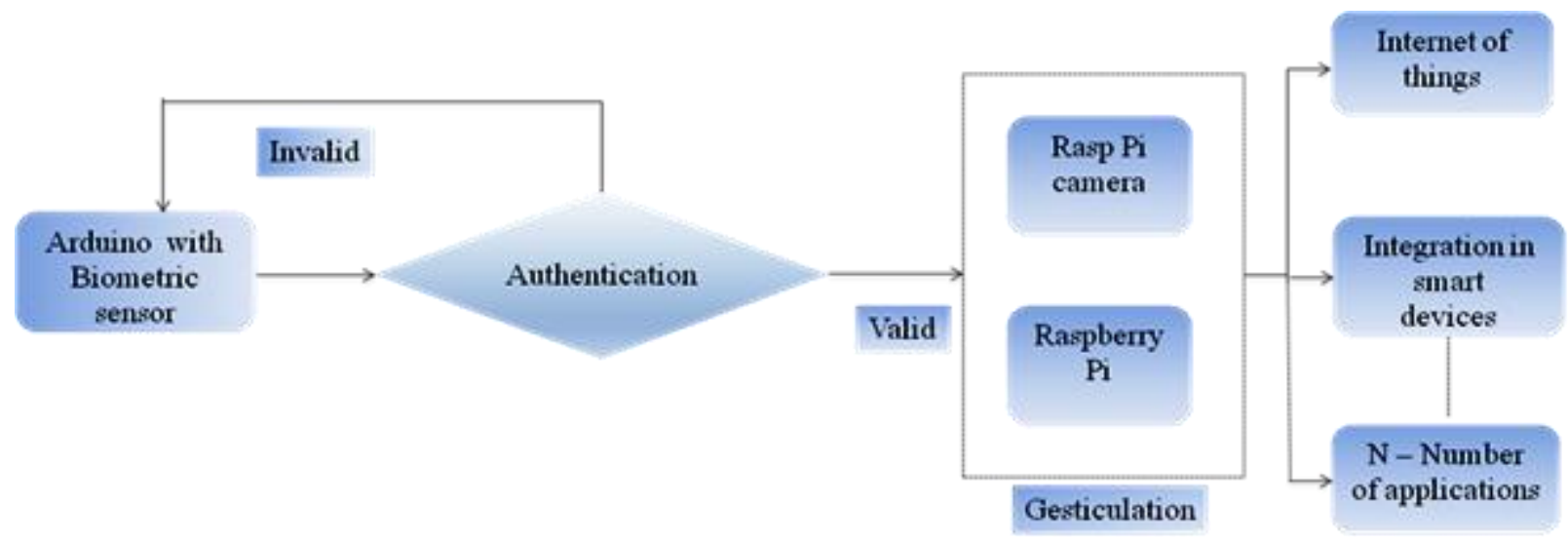

Figure 1.1- Block diagram of the gesticulation based smart surface system with bio-metric security

\section{Proposed Bio-Metric System}

The bio-metric system is implemented using Arduino. The system proposed in the figure 1.2 begins with the enrollment of the person. The finger print is kept thrice on the Biometric sensor for the proper extraction of the features. An ID is generated for every new fingerprint. The stored finger prints are then compared with the current fingerprint during the authentication stage. When the fingerprint matches the access to the Surface converter is granted. In order to enhance the security a timeout is given so that when the system is idle for longer periods, the Biometric again go backs to authentication stage.

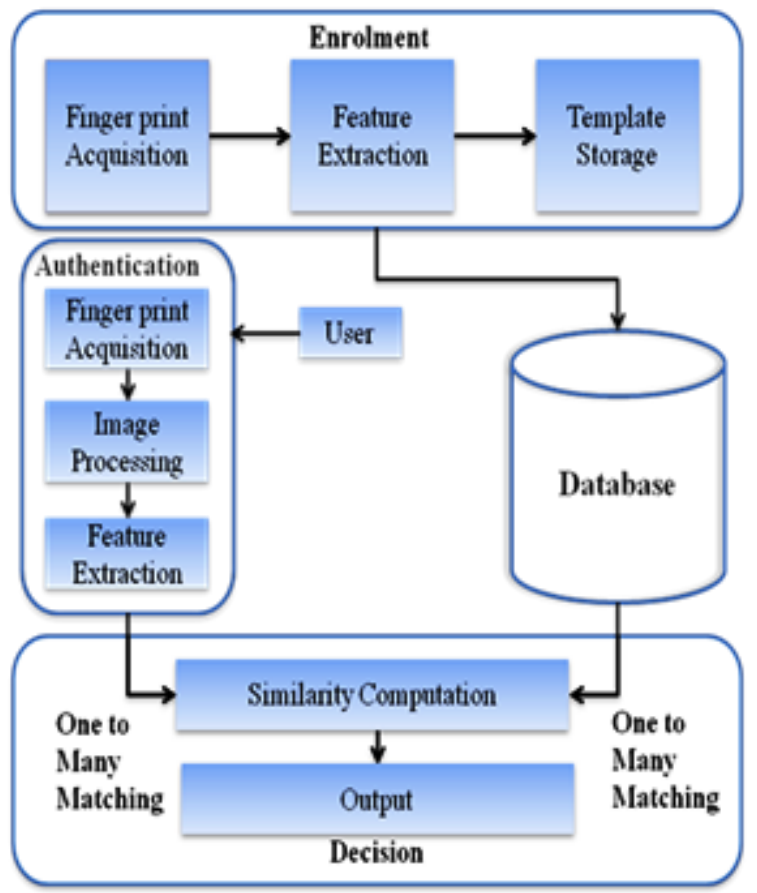

Figure 1.2 -Block diagram of the proposed Biometric system.

\section{Algorithm for bio-metric sensor:}

\begin{tabular}{|l|}
\hline Switch (choice) \\
Case 1: print ("Enrollment") \\
Get the available free ID \\
For i less than 3 \\
Capture the fingerprint \\
End for \\
Break \\
Case 2: print ("authentication") \\
Capture fingerprint \\
Compare with database \\
If (Id found) \\
Print ("access granted") \\
Else \\
Print ("access denied") \\
Break \\
End
\end{tabular}

\section{Proposed Gesture Recognition}

The figure 1.3 explains the gesture recognition is implemented using Raspberry pi. The pi 3 is used here owing to its high speed and increased RAM capacity when compared to pi 2.The operating system that is used in Raspberry pi is Raspbian Jessie with pixel 201701.The gesture recognition is carried out with a Raspi camera and Open CV 2.4.9 in Python 2.7.

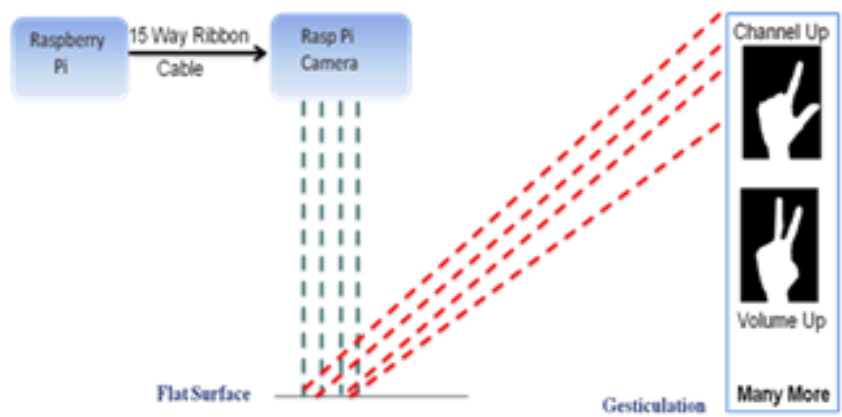

Figure 1.3- Block diagram of the proposed gesture recognition system. 
The Raspberry pi camera V2 module is used here. The Camera has a $15 \mathrm{~cm}$ cable through which it is connected with the Raspberry pi. The camera is placed in such a way that we can convert any surface into a gesture recognizing surface. The integration also requires the installation of the required libraries. The camera is enabled by using the command

\section{\$ sudo raspi-config}

Background subtraction model is used here to recognize the gestures. Open $\mathrm{CV}$ provides a number of background subtraction models among that codebook model is used here [7].The codebook model calculates the average deviation of each pixel and accordingly designates the boxes. Once the foreground image is obtained the outline of the hand is obtained using convex hull algorithm [8]. The depth points are calculated using convexity defect points.

\section{Algorithm for gesture recognition:}

$$
\begin{gathered}
\text { Cap }=\text { cv2. VideoCapture }(0) \\
\text { While }(\text { cap is opened }) \\
\text { Ret, img = cap.read }() \\
\text { Cv2.draw the rectangle } \\
\text { Grey }=\text { cv2.cvtcolour }(\text { img, }
\end{gathered}
$$

cv2.colourtogray)

$$
\text { Blurred =cv2.gaussianblur (Grey, blur, 0) }
$$

Retval, Threshold $=$ cv2.threshold (blurred,

$$
127,255 \text {, }
$$

Binaryinverted + binaryotsu

Contours, hierarchy $=($ find contour $)$

Cnt $=\max$ (contours, contourarea)

Hull $=$ cv2.convexhull (cnt)

Defects $=c v 2$. convexitydefects (cnt, hull)

Cv2.drawcontours ()

Convert the defects into tuples

Count the defects

If $($ defects $==1)$

Perform the reauired function

\section{Integration of Smart Devices}

The outputs from the GPIO pins are given to the Arduino digital pins. We can control various devices through the output from the Arduino. Here we have tried controlling Television and mouse pointer. The figure 1.4 shows the integration of the various devices. The Television is controlled using SIRC protocol. A relay is used to control the devices like lights, fans etc. Apart from the household appliances we can also use this system for controlling the mouse pointer. The mouse pointer is controlled using the visual studio software. The gestures are processed and the SIRC protocol is implemented. The IR signal is then given to the IR transmitter and that signal gets recognized by the IR receiver in Television and the required operations are performed. We can also give the signals to relay circuits.

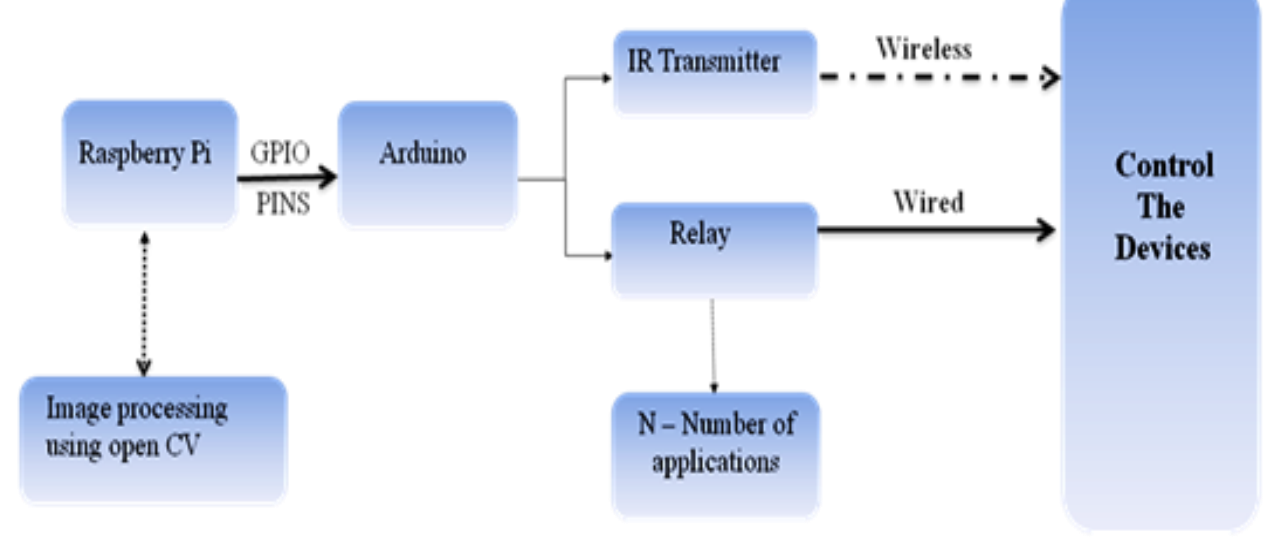

Figure 1.4- Integration of various smart devices 


\section{Hardware Implementation Result}

Figure 1.5 shows the implementation of Biometric sensor using Arduino. The Sensor captures the input and if the fingerprints are matched it sends a unique id to raspberry pi which then opens the gesture recognition software once the id is matched. Figure 1.6 explains the Raspberry pi home screen. Raspberry pi 3 is used as hardware. The OS that is used is Raspbian Jessie.Figure 1.7 represents the recognition of gesture using Open CV software. The contour image along with the threshold image is displayed in the output. The gesture when recognized will send a signal back to Arduino which will then control various devices based on the code input from Raspberry pi.

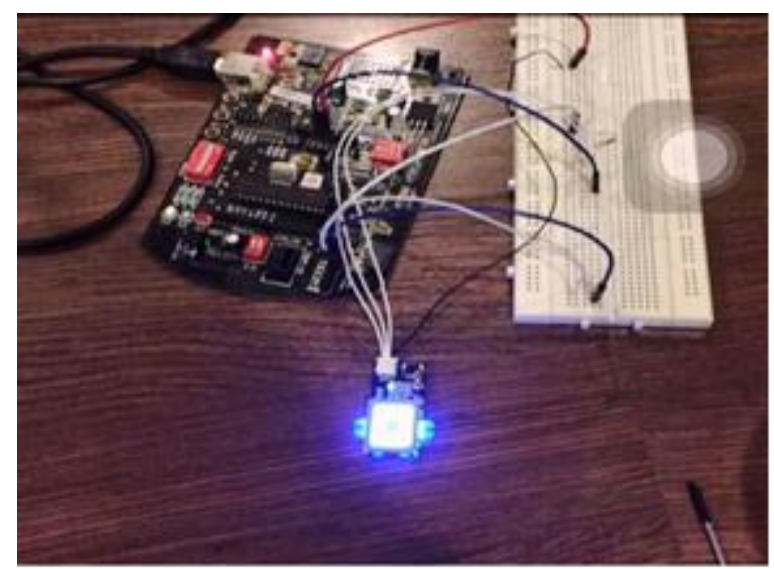

Figure 1.5 -Biometric sensor using Arduino

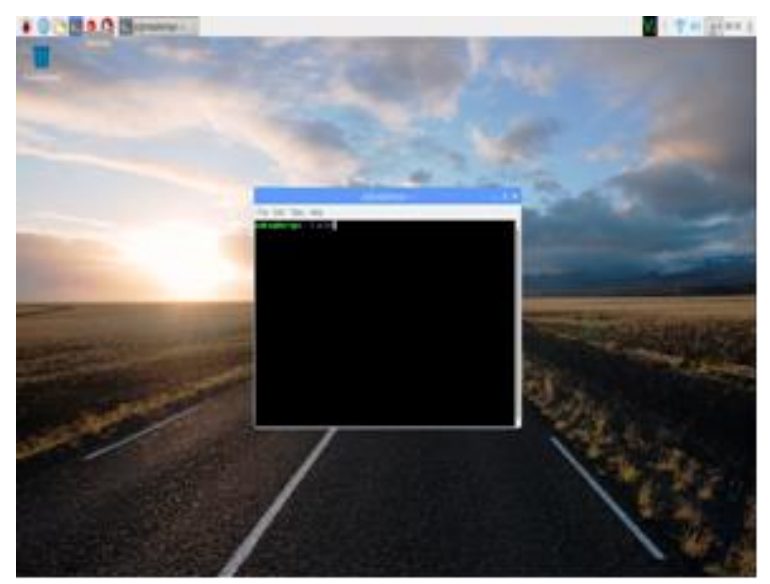

Figure 1.6- Raspberry pi home screen

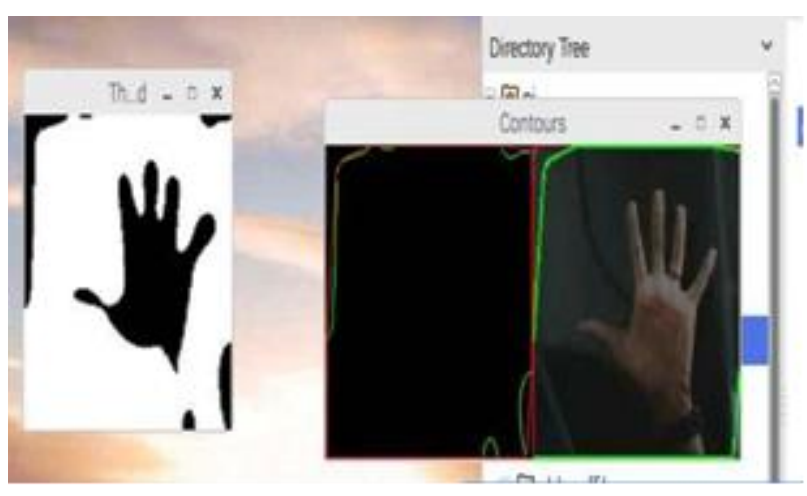

Figure 1.7-Gesture Recognition using open CV python

\section{CONCLUSION}

The proposed system controls Television and mouse pointer along with some household appliances. This concept can be integrated with all electronic appliances and the possibility of security threat is also eliminated by use of Biometrics.

\section{REFERENCES}

[1] B. Stenger, P. Mendonça, R. Cipolla, "Model based 3D tracking of an articulated hand", CVPR, Vol. 2, pp. 310- 315, 2001.

[2] Y. Wu, T.S. Huang, "Capturing natural hand articulation", Proc. 8th Int. Conf. on Computer Vision, Vol. 2, pp. 426-432, 2001.

[3] N. Gupta, P. Mittal, Y. Sumantra, D. Roy, S. Chaudhury, and S. Banerjee, "Developing a gesturebased interface,"IETE Journal of Research, Vol. 48, pp. 237-244, 2002.

[4] J.R. New, E. Hasanbelliu, M. Aguilar, "Facilitating User Interaction with Complex Systems via Hand Gesture Recognition", Proceedings of the 2003 Southeastern ACM Conference, 2003.

[5] K. Gill, S.-H. Yang, F. Yao, X. Lu, “A ZigBeebased home automation system", IEEE Trans. Consum. Electron. Vol. 55, no. 2, pp. 422-430, 2009.

[6] R.Gayathri and P. Ramamoorthy, "Performance Evaluation of Multimodal Multifeature Authentication System Using KNN Classification," The Scientific World Journal, vol. 2015, Article ID 762341, 9 pages, 2015. doi:10.1155/2015/762341

[7] K. Kim, T. Chalidabhongse, D. Harwood, L. Davis, "Background modeling and subtraction by codebook construction", Proc. IEEE Int. Conf. Image Process., Vol. 5, pp. 3061-3064, 2004. 
[8] H. R. Khosravani, A. E. Ruano, and P. M. Ferreira, "A simple algorithm for convex hull determination in high dimensions", in Intelligent Signal Processing (WISP), IEEE 8th International Symposium on. IEEE, pp. 109-114, 2013.

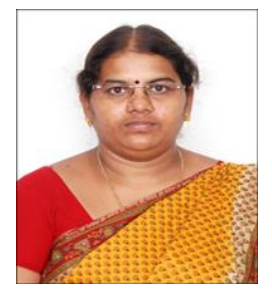

R.Gayathri received B.E degree in Electronics and Communication Engineering from Madras University, Master's and doctoral degree from Anna University, College of Engineering Guindy, Chennai, India in 1999, 2001 and 2014 respectively. She is currently working as Associate Professor in the Department of Electronics and Communication Engineering, Sri Venkateswara College of Engineering, Affiliated to Anna University, Chennai, India. She has 19 years of experience in teaching and research. She has published numerous high impact journals and her research work has received hundreds of citations. She has handled multitude subjects and right now guiding $12 \mathrm{Ph} . \mathrm{D}$. scholars. She is extending her contribution to both academics and research by being doctoral committee member to many scholars and reviewing multitude international journals. Her research interest includes pattern recognition, computer vision, machine learning, application to image recognition, network security.

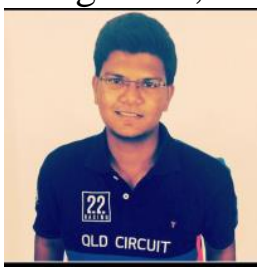

E.Roshith is a member of IETE. He is an under graduate scholar in the department of Electronics and Communication Engineering, Sri Venkateswara College of Engineering, Affiliated to Anna University, Chennai. His research interest includes pattern recognition, computer vision, machine learning, application to image recognition, network security.

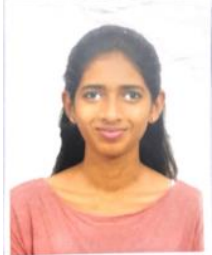

B.Sanjana is a member of IETE. She is an under graduate scholar in the department of Electronics and Communication Engineering, Sri Venkateswara College of Engineering, Affiliated to Anna University, Chennai. Her research interest includes pattern recognition, computer vision, network security

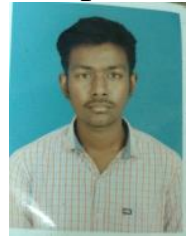

S. Sanjeev Kumar is a member of IETE. He is an under graduate scholar in the department of Electronics and Communication Engineering, Sri Venkateswara College of Engineering, Affiliated to Anna University, Chennai. His research interest includes machine learning, application to image recognition, network security.

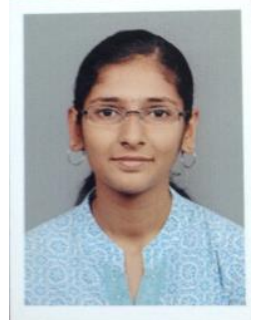

R.Shyamala is a member of IETE. She is an under graduate scholar in the department of Electronics and Communication Engineering, Sri Venkateswara College of Engineering, Affiliated to Anna University, Chennai. Her research interest includes pattern recognition, computer vision. 